\title{
A Survey on Application of Machine Learning to Resource Management in Grid Environment
}

\author{
Susmita Singh, Madhulina Sarkar, Sarbani Roy, and Nandini Mukherjee
}

\begin{abstract}
Grid represents an environment over a distributed area, incorporating heterogeneous elements such as server nodes, storage devices, and network components in a scalable, wide-area spanning compute infrastructure. Since a Grid requires large scale resource sharing, efficient resource management system (RMS) is required to manage the Quality of Service (QoS). One of the chief tasks of an RMS for Grid is selecting an appropriate and most suitable resource provider for execution of a particular job submitted by a user. This is also known as resource brokering. This paper presents a survey of resource brokering paradigms based on Machine Learning (ML). Three major ML techniques applied in Grid resource management for this purpose have been discussed.
\end{abstract}

Index Terms-Grid computing, resource management, machine learning, resource brokering, load balancing.

\section{INTRODUCTION}

Since a computational Grid [1], [2] focuses on large scale resource sharing, resource management is crucial to its operations [3]. However, this is a complicated task as the Grid environment is highly dynamic and involves multiple organizations having different sets of policies. The size of a Grid may be very large, potentially scalable to the Internet size. This large Grid or network computing system can be viewed as a virtual computer consisting of a networked set of heterogeneous machines that agree to share their local resources with each other.

A Grid can have many users each with their own set of jobs to be submitted to the Grid for execution. The jobs should be allocated to resources in such a way that the workload is balanced across all the resource providers. Whenever a job is submitted to the Grid, the RMS should find an appropriate resource provider where it can be scheduled without violating the service level agreements or Grid policies. An efficient RMS must be able to perform its operation in a reasonable amount of time and with an acceptable accuracy in predicting the best resource for a given job. It should also take load balancing into account.

To achieve this goal, ML techniques have been found to be very useful. ML systems have a basic characteristic of

Manuscript received February 4, 2013; revised April 11, 2013. This work was supported by the Department of Computer Science and Engineering, Jadavpur University,Kolkata, India.

S. Singh, S. Royc, and N. Mukherjee are with the Department of Computer Science and Engineering, Jadavpur University, Kolkata, India (e-mail:_susmitasingh09@gmail.com,_sarbani.roy@ieee.org, numkherjee@cse.jdvu.ac.in).

M. Sarkar is with the Department of Computer Science and Engineering, Government College of Engineering and Leather Technology, Kolkata, India (e-mail: madhulina.sarkar@gmail.com). learning from examples and adapting themselves according to environment. This especially suits the Grid environment since the system is highly dynamic and multi-organizational. This paper presents a brief survey of application of three efficient ML techniques - fuzzy decision tree learning, fuzzy neural network learning and genetic algorithms to Grid resource brokering.

\section{Motivation}

ML [4] is the study of design and development of algorithms to make computers learn from experience and use this knowledge for better results of a given problem. The problem is often classification of data into various clusters or identification of patterns in data. We can say ML is used for searching through a model space. A Grid RMS aims to produce optimal job-resource combinations so that the execution time or cost of jobs submitted by a user is minimized and $\mathrm{QoS}$ is maintained. Therefore, the work of RMS can be viewed as an extensive search through the space of various possible allocations of jobs to resources. So, ML techniques are suitable for this.

One of the chief advantages of ML techniques over other methods is their low time complexity. ML search techniques are much more efficient than the computationally intractable exhaustive search of all possible combinations. Secondly, no human intervention is required. The search process is automatic. This saves human resources. Another advantage of ML techniques is consistency of results. A good and trained ML system gives around $80 \%$ accuracy in finding the optimal (or very near to optimal) solution for a given problem [5].

Grid environment is highly dynamic with resources being constantly added and removed. The users must remain unaware of scheduling details and see the entire system as a virtual one. For this, the RMS has to do the scheduling autonomously and in time efficient manner with no human intervention. As discussed above, ML techniques seem promising as they ensure these requirements.

\section{ISSUES IN APPLYING ML TO GRID RMS}

ML systems give good results once they have been initially trained or customized for the desired purpose. But the training (in case of supervised learning) and customization (finding appropriate system parameters in case of unsupervised learning) is a challenging task [4].

In our survey paper, we have considered decision tree [6], neural network [7] and GA [8] for resource management.

We will describe the various issues and challenges in using 
these three techniques.

\section{A. Decision Tree Learning}

The reliability of the information in the decision tree depends on feeding the precise internal and external information at the onset. Even a small change in input data can at times, cause large changes in the tree. Another fundamental flaw of the decision tree analysis is that the decisions contained in the decision tree are based on expectations, and irrational expectations can lead to flaws and errors in the decision tree.

\section{B. Neural Networks}

Neural networks' efficiency depends a lot on its design. Improper selection of layout of perceptrons, number of layers or thresholding functions may lead to poor output. They are also prone to the problems of over-fitting. This is the situation when the network can classify training data with high accuracy but test data is not classified properly. This arises out of wrong selection of training examples.

\section{Genetic Algorithms}

In GA, the prospective solutions are modelled using chromosomes. A fitness function evaluates the fitness of these chromosomes which indicates how good the solution is. Thus, the selection of appropriate fitness function is of critical importance. The chromosomes have to be designed in such a manner that all important characteristics of problem under consideration are represented but the length is not too long.

\section{ML BASED GRID RMS}

This section gives a brief description of three modern approaches using ML techniques to Grid resource management in the aspects of resource brokering and load balancing. These techniques are fuzzy decision tree (FDT) based learning; fuzzy neural network (FNN) based learning and GA.

\section{A. FDT Based Resource Management}

Induced decision trees [9] are an extensively researched solution to classification tasks. General decision tree always has a deterministic result. This feature is unsuitable for certain applications. We may need to model real life problems more closely where the data often has inherent uncertainty or fuzziness. Fuzzy logic [10] enables better description of such situations. Decision trees be built on fuzzy data are known as FDT. [11], [12]. In case of an FDT, the attributes at nodes are fuzzy variables. In case of FDT, we deal with ranges of membership function values instead of actual data values.

Asgarali et al., [13] proposed a resource broker architecture based on FDT learning. The role of this broker service is using learning method to find the best node according to requirements of the job and distributed computing resources of the Grid. This application can be executed on the top of Globus toolkit middleware. In this architecture, first an FDT is constructed using the FDT Algorithm [14], [15]. This algorithm is a developed version of ID3 [16] that operates on fuzzy sets and produces an FDT.
Then, the incoming new sample is classified according to the label of the leaf node with maximum confidence (fuzzy membership) or fitness value. The general architecture of the system consists of various components which are described as follows:

Nodes represent the various resource providers present in the Grid environment. The result of every node is sent in an XML document and is stored in a temporary XML database (TXD).

Miner application: Each node is attached with a miner application (MA) that maintains a log file. This log file serves as a small internal database. Whenever a node is connected to the Grid or a new job is submitted to the node, its MA updates the $\log$ file by inserting a new record in the database indicating the type of activity. The other fundamental task of MA is to receive and process packets sent by the Grid resource broker (GRB) during node selection process. If there are many nodes in the network that are ready for executing jobs and the broker wants to select some nodes from this pool of nodes, it sends a packet to each connected node. This packet contains some information about a new job (like IP address of sender, size of job, size of RAM needed, average time needed for execution, approximate execution start time, minimum power of CPU etc.). On the other hand, when the MA on the destination node receives this packet, it analyzes the information contained in it. If there are sufficient resources to do the job, MA will perform a data processing operation on its own log file to obtain various parameters of the node like average hit ratio, number of submitted jobs on this node, average response time for finished jobs etc. These results, along with resource characteristics of the node, are sent to the GRB. Then the GRB analyzes them to select or refuse the desired node.

Broker layer: This layer consists of three modules - the general broker, request broker and resource selector. The request broker module broadcasts packets to all nodes in the Grid to request their characteristics and parameters. Then it must receive and save the sent results from each node in the TXD. The latest sent results are kept in this database. Next, the resource selector section will execute a FDT algorithm on TXD (that is, on the gathered results) to obtain the FDT. This task is done in a sub-section inside resource selector called FDT executor. When this algorithm has finished its task, the next sub-section, SNJ (selecting node for job) uses the result of this algorithm, that is, the FDT, to identify suitable nodes.

In the FDT based architecture of [13], the costliest step is building up the FDT. An increase in the number of attributes or training examples causes an increase in cost but this makes resource selection more accurate. Once the FDT has been constructed, classifying the job-resource combinations using it is simple and of logarithmic complexity with respect to number of nodes in the tree. The FDT based architecture of [13] has a performance bottleneck at centralized broker where the Fuzzy DC Executer and SNJ units are located. The request broker has to send packets to all the resource providers in the Grid and wait for their responses in order to get a correct system snapshot. The broker application runs at a centralized server only.

\section{B. FNN Based Resource Management}

An FNN [17], [18] is an ANN where the inputs and outputs 
are fuzzy variables.

Kun-Ming Yu et al., [19] proposed a high performance algorithm based on FNNs to solve the otherwise intractable problem of job scheduling in Grid environment. In this algorithm, fuzzy logic [10] is used to evaluate the Grid system load status and neural network [7] is used to automatically tune the membership functions. It has been implemented on top of Globus toolkit 4 middleware for verification of performance of the proposed scheduling algorithm.

Grid environment in which this approach is applied consists of three hierarchical tiers: resource broker tier, Grid head tier and computing tier.

Resource broker tier: This tier is comprised of many components and acts as the coordinator between users and resource providers. Two components have been functionally grouped into two modules - kernel module and load balancing module.

\begin{tabular}{|c|c|c|c|}
\hline $\begin{array}{l}\text { CPU } \\
\text { Memory }\end{array}$ & Light & Medium & Heavy \\
\hline Light & Very Light & Medium & Heavy \\
\hline Medium & Light & Medium & Heavy \\
\hline Heavy & Medium & Heavy & Very Heavy \\
\hline
\end{tabular}

Kernel module: It consists of a command line user interface, an Execution Manager to execute jobs generated from user's requirements, Transfer Manager to transfer files and related data and Information Manager to collect information from the computing resources.

Load balancing module: Its aim is to accurately measure the workload status of each node in the Grid. The load balancing module consists of fuzzy logic workload measurement and neural network training components.

Fuzzy logic workload measurement: The workload measurement technique using fuzzy logic described in [20] has been used in [19]. CPU utilization and memory utilization have been considered the input fuzzy variables. Both of them were normalized and characterized by the membership functions Light, Heavy and Medium. Then, the following 9 inference rules shown in Table I were applied to calculate the workload of each node. Max-min inference method and center of gravity defuzzification are used to calculate workload.

Neural network training component: It is used to self organize and tune the membership functions defined above. The neural net consists of five layers - an input layer, three hidden layers and an output layer. The operations of this component include feed forward phase and back propagation phase. In feed forward phase, the workload information in form of CPU utilization and memory utilization is given as input to the Input layer. The input values are then individually fuzzified using three fuzzy sets Light, Medium and Heavy in the first hidden layer. The second hidden layer displays the nine fuzzy inference rules applied in the system which are shown in Table I. The neurons in this layer compute the minimum value of the fuzzified degrees received from the first layer, which indicates degree of match for the condition part of the table. These minimum values are then sent to the linked neuron in the third hidden layer and computed by the maximum operator to complete the max-min rule inference process. The output layer calculates the workload degree by centre of gravity defuzzification. In back propoagation phase, the tolerance value of each node is set. This is done once for every five times of feed forward in order to reduce training overhead.

Grid head tier: This tier is responsible for submitting jobs to the corresponding computing nodes and communicating with the resource broker.

Computing tier: This tier is composed of the actual nodes or resources of the Grid. These nodes are responsible for executing jobs. The nodes are grouped into clusters each supervised by one Grid head.

The FNN based architecture described in [19] requires training of the neural network for workload measurement. This cost is linear with respect to the product of total number of fuzzy sets of all input variables and the number of nodes in the second hidden layer. There is another cost component associated with back propagation phase. It is linear with respect to the number of nodes in second and third hidden layer. Detecting an inference rule and workload calculation using this neural network is fast and has constant time complexity. The FNN based architecture of [19] also has a centralized broker tier. However, the task of resource discovery is hierarchically distributed among the Grid heads. The workload measurement and tracking task is further distributed among all the resources as a workload observer is attached in computing tier. Thus, the system faces a problem of bottleneck at the centralized broker where the neural net calculates load for all resource providers. But it is not very pronounced as load is mostly balanced and other tiers participate as mentioned.

\section{GA Based Resource Management}

GAs [21], [8] are function optimization or search heuristics that try to imitate the natural evolution process using selection, mutation and crossover operators. In a GA, a population of strings called chromosomes encode candidate solutions called to an optimization problem. The population evolves toward better solutions. Traditionally, solutions are represented in binary as strings of $0 \mathrm{~s}$ and $1 \mathrm{~s}$, but other encodings may be needed depending on problem specification. The evolution usually starts from a population of randomly generated individuals and happens in generations. In each generation, the fitness of every individual in the population is evaluated using a fitness function, multiple individuals are stochastically selected from the current population based on their fitness, and modified using crossover and mutation operators to form a new population. The new population is then used in the next iteration of the algorithm. Commonly, the algorithm terminates when either a maximum number of generations has been produced, or a satisfactory fitness level has been reached for the population. Generally, a satisfactory and near-optimal solution is reached within a much shorter time than exhaustive search of solution space. 
Javier Carretero et al., [22] proposed a GA based schedulers for efficient allocation of jobs to Grid resources. The aim of these schedulers is to minimize the makespan and flowtime of the jobs in the Grid. Makespan is the time when the last job is finalized and flowtime is the sum of finalization times of all the jobs. A number of schedules have been developed and implemented in $\mathrm{C}++$ by using different GA operators and encoding schemes. The Expected Time to Complete (ETC) model [23][24] has been adopted. This assumes that we know the computing capacity of each resource, and can estimate or predict the workload information of each job and the load of prior work of each resource. These data are either provided by the user, or found from historic data or predicted.

The GA based architecture described in [22] has a variable operating cost depending upon the execution of GA and selection of operators. The cost incurred is a product of combined costs of applying various operators and the number of runs of GA. Most of the operators are fast and linear with respect to chromosome length, that is, the number of job and resource specification parameters. The results obtained using GA are mostly satisfactory especially if elitist approach is used and time complexity is much lesser compared to exhaustive search in case of optimization problems. The GA based architecture of [22] involves generation of population and application of evolution operators. Initial population size is constant and not very large. Thus, initialization can be done at a central server without posing a significant bottleneck. The task of application of crossover and mutation operators can be distributed among many resources if the population size is large. However, for small populations, it is more efficiently done at the central server. The broker may also be distributed in a sense if each generation is handled on a different resource provider

\section{DISCUSSION}

The architectures described above incorporate ML successfully into the Grid environment. But there are some issues that need to be handled by an efficient RMS in a Grid environment which are not addressed properly by any of these. We will now discuss some of these issues.

\section{A. $Q o S$}

Any Grid system is expected to maintain a certain QoS for each job as per requirements of the user [25]. QoS is maintained through Service Level Agreements between clients and resource providers. The RMS should set up and maintain these SLA for each user. Any of the above architectures does not consider the specification of QoS for any job.

\section{B. Dynamicity of Workload}

The workload in a Grid environment is highly dynamic. Resources, users and jobs may be added deleted quite frequently. The above architectures make resource brokering decisions based on a current snapshot, that is, a particular configuration of resources, users and jobs, of the Grid. They do not take snapshots dynamically based on the current workload but statically at fixed intervals of time.

\section{Maintenance of Historical Data}

An ML based Grid RMS learns from the examples. If the data about already scheduled jobs is stored in a database, it can act as an example repository for the learning system. This data includes which job was scheduled to which resource provider and what the resource provider's configuration was at that time. None of the above architectures has provision for automatic recording and storage of such historical data.

\section{Load Balancing}

In a Grid environment, it is desirable to have a balanced workload distribution across the resource providers. This ensures optimal performance and a sound multi-organizational policy. Among the architectures discussed above, only the FNN based resource broker [19] addressed the issue of load balancing while the other two have not taken load balancing into consideration for brokering decisions.

To address the above issues, we suggest a new RMS architecture based on supervised GA. The supervision will be obtained from previous job schedules stored in the database. The database will be distributed across the Grid to avoid performance bottleneck at a single resource provider. A similarity measure will be defined based on job characteristics to determine similarity between two jobs. This will be used when a new job arrives which is close but not exactly similar to some job whose data is stored in the database. For totally new kind of job, the resource selection will be based entirely on calculation of some compatibility measure based on job requirements and resource characteristics. QoS support is another significant issue in Grid environments. QoS will be explicitly specified for each job based on an SLA. Whenever a resource provider is considered for a job, it will be ensured that QoS is maintained for that job. QoS policies will be designed for this purpose. The dynamicity of workload will be considered during brokering decisions by defining a dynamic snapshot-taking policy. The snapshots will be taken at regular intervals of time but this interval will be adjusted every time there is a significant change in system workload. The interval will be short when system workload is high and long when the workload is low. We will also consider load balancing during brokering decisions and define policies to calculate and equalize workload on all resource providers.

\section{CONCLUSION}

This paper presented an overview of the motivation and challenges of using ML algorithms for resource brokering in Grid computing, followed by a survey of three modern resource-broker architectures based on ML techniques of fuzzy decision tree, fuzzy neural network and GAs. It contributes in giving an idea of how resource brokers can incorporate ML for brokering process and also helped to identify some of the key ML based Grid resource management approaches and issues that are yet to be explored as topics of future research. Then we presented a discussion on their limitations with respect to maintenance of QoS, historical data, handling dynamicity of workload and load balancing and suggested the design of a new ML based 
RMS which will overcome these limitations.

\section{REFERENCES}

[1] I. Foster and C. Kesselman, The Grid: Blueprint for a New Computing Infrastructure, San Francisco, CA: Morgan Kaufmann, 1999.

[2] I. Foster. C. Kesselman, and S. Tuecke, "The anatomy of the grid: enabling scalable virtual organizations," Int. J. Supercomput. Appl., vol 15 , no. 3, pp. 200-222, 2001

[3] K. Krauter, R. Buyya, and M. Maheswaran, "A taxonomy and survey of Grid resource management systems," Int. J Softw.: Pract. Exp., vol. 32, no. 2, pp. 135-164, 2002.

[4] Y. C. Jin and B. Sendhoff, "Pareto-based multiobjective machine learning: An overview and case studies," IEEE Transactions on Systems, Man and Cybernetics, part C: Applications and Reviews, vol. 38 , issue 3, pp. 397-415, 2008.

[5] Website Information. [Online]. Available: $\mathrm{http}: / /$ ppt.cmclre.com/m/machine-learning-ml-classification-asis-t-theamerican-society-w347-ppt.ppt.

[6] A. Shamim, H. Hussain, and M. U. Shaikh, "A framework for generation of rules from decision tree and decision table," in Proc. International Conference on Information and Emerging Technologies, 2010, pp. 1-6.

[7] K. W. Yue, U. H. Zhou, C. Cheng, J. Yang, Z. Y. He, and N. Liang, "Research on smart grid power quality assessment based on RBF neural networks and accelerating genetic algorithms," in Proc. International Conference on Advanced Power System Automation and Protection, vol. 3, 2011, pp. 2036-2039.

[8] P. F. Guo, X. Z. Wang, and Y. S. Han, "The enhanced genetic algorithms for optimization design," in Proc. IEEE $3^{\text {rd }}$ International Conference on BMEI, vol. 7, 2010, pp. 2990-2994.

[9] J. R. Quinlan, "Induction of decision trees," Centre for Advanced Computing Studies, 1985.

[10] G. J. Klir and B. Yuan, Fuzzy sets and fuzzy logic: Theory and applications, Prentice Hall, 1995.

[11] X. Z. Wang, J. H. Zhai, and S. F. Zhang, "Fuzzy decision tree based on the important degree of fuzzy attribute," vol. 1, pp. 511-516, in Proc IEEE International Conference on Machine Learning and Cybernetics, 2008.

[12] M. A. Elashiri, H. A. Hefny, and A. H. Elwahab, "Introduction of fuzzy decision trees based on fuzzy rough set techniques," in Proc. IEEE International Conference on Computer Engineering and Systems, 2011 , pp. 134-139.

[13] A. Bouyer, M. Karimi, M. Jaleli, and M. D. Mohd. Noor, "A new approach for selecting best reousrce nodes by using fuzzy decision tree in grid resource broker," International Journal of Grid and Distributed Computing, 2006.

[14] M. Umano, H. Okamoto, H. Tamura, F. Kawachi, S. Umedzu, and J. Kinoshita, "Fuzzy decision trees by fuzzy ID3 algorithm and its application to diagnosis systems," Department of Systems Engineering and Precision Engineering, Osaka University, Japan, IEEE, 1994.

[15] C. Marsala and B. B-Meunier, "Choice of a method for the construction of fuzzy decision trees," The IEEE International Conference on Fuzzy Systems, University P. e M. Curie, Paris, 2003.

[16] X. Y. Shao, G. J. Zhang, P. G. Li, and Y. B. Chen, "Application of ID3 algorithm in knowledge acquisition for tolerance design," Journal of Materials Processing Technology, vol. 117, issue 1-2, pp. 66-74, 2001

[17] P. M. Birgani, M. Ashtiyani, and S. Asadi, "MRI segmentation using Fuzzy c-means clustering algorithm basis neural network," in Proc. $3^{\text {rd }}$ International Conference on ICTTA, 2008, pp. 1-5.

[18] X. F. Dai, L. N. Zhao, and H. Zhang, "Local map matching based on fuzzy neural networks for hierarchical SLAM," in Proc. IEEE $7^{\text {th }}$ International Conference on FSKD, vol. 2, 2010, pp. 744-747.

[19] K. M. Yu, Z. J. Luo, C. H. Chou, C. K. Chen, and J. Y. Zhou, A fuzzy neural network based scheduling algorithm for job assignment on computational grids, Springer-Verlag Berlin Heidelberg, 2007.

[20] J. Zhou, K. M. Yu, C. H. Chou, L. A. Yang, and Z. J. Luo, "A dynamic resource broker and fuzzy logic based scheduling algorithm in grid environment," presented at International Conference on Adaptive and Natural Computing Algorithms, LNCS, Springer, Heidelberg, 2007.

[21] K. Waqas, R. Baig, and S. Ali, "Feature subset selection using multiobjective genetic algorithms," in Proc. IEEE $13^{\text {th }}$ International Multitopic Conference, 2009, pp. 1-6.
[22] J. Carretero and F. Xhafa, "Genetic algorithm based schedulers for grid computing system," International Journal of Innovative Computing, Information and Control, University of Politenica de Catalunya, Spain, Ajith Abraham, vol. 3, no. 6, December 2007.

[23] M. Maheswaran, S. Ali, H. J. Siegel, D. Hensgen, and R. F. Freund, "Dynamic mapping of a class of independent tasks onto heterogeneous computing systems," Journal of Parallel and Distributed Computing, vol. 59, no. 2, pp. 107-131, 1999 .

[24] H. J. Braun, T. D. Siegel, N. Beck, L. L. Blni, M. Maheswaran, A. I. Reuther, J. P. Robertson, M. D. Theys, and B. Yao, "A comparison of eleven static heuristics for mapping a class of independent tasks onto heterogeneous distributed computing systems," Journal of Parallel and Distributed Computing, vol. 61, no. 6, pp. 810-837, 2001.

[25] S. Roy and N. Mukherjee, "Efficient resource management for running multiple concurrent jobs in a computational grid environment," Journal of Future Generation Computer Systems, vol. 27, issue 8, pp. 1070-1082, 2011.

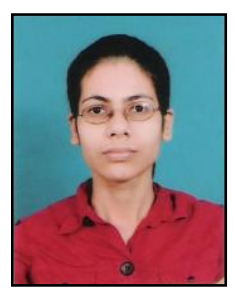

Susmita Singh is a second year Master of Computer Science and Engineering student at Jadavpur University, Kolkata, India. She received B.Tech degree in Computer Science and Engineering from Techno India, Kolkata, West Bengal, India under West Bengal University of Technology in 2011.

She has successfully completed projects titled "Demonstration of Regression Testing for Object-oriented Programs" at the Indian Institute of Technology, Kharagpur, West Bengal, India in June 2010 and "Development of a Secure Hierarchical Routing Protocol for Wireless Sensor Networks" at Techno India, Kolkata, West Bengal, India in September 2010- May 2011 She has worked as an Intern in Codez IT Solutions, Kolkata, West Bengal, India in July-August 2010 in the field on web application development on .NET platform. Her areas of interest are Grid Computing, Machine Learning and Wireless Sensor Networks.

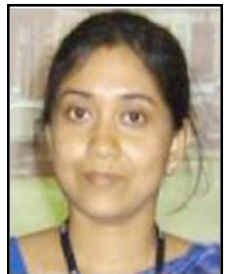

Madhulina Sarkar received M.Tech degree in Computer Technology from Jadavpur University, Kolkata, West Bengal, India in 2007 and B.Tech degree in Computer Science from University of Kalyani, Kalyani, West Bengal, India in 2003.

She has been working as a Faculty Member in the department of Computer Science and Engineering, Government college of Engineering and Leather Technology, Kolkata, West Bengal, India since 2009. Her areas of interest are Grid Computing and Distributed Computing.

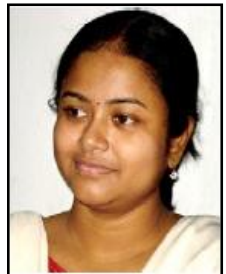

Sarbani Roy received Ph.D. degree in Engineering from Jadavpur University, Kolkata, West Bengal, India in 2008, and M.Tech degree in Computer Science and Engineering, M.Sc. degree in Computer and Information Science and B.Sc. Honors degree in Computer Science from Calcutta University, Kolkata, West Bengal, India in 2002, 2000 and 1998 respectively.

She has been working as a FACULTY MEMBER in the department of Computer Science and Engineering, Jadavpur University, Kolkata, West Bengal, India since 2006. Her areas of interest are Grid and Cloud Computing, Distributed Computing, Wireless Sensor Networks and Mobile Computing.

She is a member of IEEE.

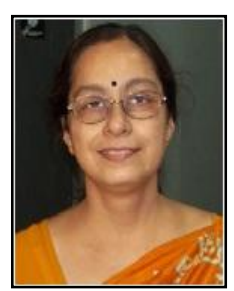

Nandini Mukherjee joined as a Faculty Member in the Department of Computer Science and Engineering, Jadavpur University, Kolkata, West Bengal, India in 1992. In 1996, she was awarded a Commonwealth Scholarship, tenable in the UK. She completed her $\mathrm{Ph} . \mathrm{D}$ in Computer Science from the University of Manchester, UK in 1999. She became a PROFESSOR at Jadavpur University in 2006. Currently, she is the director of the School of Mobile Computing and Communication, Jadavpur University. Her research interest areas are High Performance Parallel Computing, Grid Computing and Mobile Computing. She is a senior member of IEEE. 